\title{
Vectorial theory of propagation in uniaxially anisotropic media
}

\author{
Alessandro Ciattoni, Bruno Crosignani, and Paolo Di Porto \\ Dipartimento di Fisica, Università dell'Aquila, 67010 L'Aquila, Italy, \\ and Istituto Nazionale di Fisica della Materia, Unità di Roma "La Sapienza," 00185 Roma, Italy
}

Received August 4, 2000; revised manuscript received November 23, 2000; accepted December 13, 2000

\begin{abstract}
We describe propagation in a uniaxially anisotropic medium by relying on a suitable plane-wave angularspectrum representation of the electromagnetic field. We obtain paraxial expressions for both ordinary and extraordinary components that satisfy two decoupled parabolic equations. As an application, we obtain, for a particular input beam (a quasi-Gaussian beam), analytical results that allow us to identify some relevant features of propagation in uniaxial crystals. (C) 2001 Optical Society of America
\end{abstract}

OCIS codes: $260.1180,260.1960$.

\section{INTRODUCTION}

Electromagnetic propagation in anisotropic media ${ }^{1,2}$ is still receiving a good deal of attention from both the experimental and the theoretical points of view. In particular, the vectorial nature of light traveling through an anisotropic crystal plays a relevant role and cannot be neglected when designing polarizers and compensators that are aimed at obtaining a given polarization state of optical radiation. Besides, anisotropic materials play a central role in amplitude- and phase-modulation devices ${ }^{3}$ and in nonlinear optical phenomena, as, e.g., secondharmonic generation and the photorefractive effect. ${ }^{4} \mathrm{Op}$ tical propagation in uniaxially anisotropic media has been investigated in connection with fields radiated by a finite source in an unbounded uniaxial crystal under very general conditions (nonmonochromatic fields, dispersive materials, and arbitrary propagation direction) by reliance on the plane-wave angular spectrum..$^{5}$

In this paper, we straightforwardly derive the planewave angular spectrum of the superimposed ordinary and extraordinary monochromatic plane waves that are present inside a uniaxial crystal. This approach allows us to solve, in principle, the boundary-value problem, that is, to obtain the field in the half-space $(z>0)$ after its distribution is known on the reference plane $(z=0)$. In agreement with birefringence theory an arbitrary field distribution for $z=0$ is found to give rise to ordinary and extraordinary components that independently propagate through the medium. Our formalism allows us to develop a paraxial theory of propagation (to deal with beams whose transverse dimension is much larger than a wavelength), giving rise to two decoupled parabolic equations for ordinary and extraordinary components that generalize the standard treatment of isotropic paraxial theory. In particular, their general solutions for vectorial fields are specialized to obtain exact analytical results for a class of input beams that possess a Gaussian shape with a small hump on the side. This analytical description reveals relevant features, such as the progressive change of polarization of the field, of propagation in anistropic media.
The paper is organized as follows: In Section 2, we obtain the plane-wave angular-spectrum representation of a vectorial monochromatic optical beam. In Section 3, we deduce paraxial expressions for both the ordinary and the extraordinary components of the field and for the two decoupled parabolic equations that they obey. In Section 4, we specify our formalism to derive an explicit analytical solution.

\section{ANGULAR-SPECTRUM REPRESENTATION OF A MONOCHROMATIC FIELD IN A UNIAXIAL CRYSTAL}

The complex amplitude $\mathbf{E}(\mathbf{r})$ of the field $\mathbf{E}(\mathbf{r}, t)$ $=\operatorname{Re}[\mathbf{E}(\mathbf{r}) \exp (-i \omega t)]$ propagating in an anisotropic medium obeys an equation that is completely equivalent to Maxwell's equations and reads as

$$
\nabla^{2} \mathbf{E}-\nabla(\nabla \cdot E)+k_{0}^{2} \boldsymbol{\epsilon} \cdot \mathbf{E}=0
$$

where $k_{0}=\omega / c$ and $\epsilon$ is the relative dielectric tensor. For a uniaxially anisotropic crystal, we can write

$$
\epsilon=\left[\begin{array}{ccc}
n_{o}^{2} & 0 & 0 \\
0 & n_{o}^{2} & 0 \\
0 & 0 & n_{e}^{2}
\end{array}\right],
$$

where $n_{o}$ and $n_{e}$ are the so-called ordinary and extraordinary refractive indices, respectively, of the crystal, as we assume that the $z$ axis coincides with the optical axis. In the following, we consider propagation along the $z$ axis, although our formalism can be extended to an arbitrary propagation direction, this being the geometry most often encountered in practice.

The fundamental solutions of Eq. (1) are two sets of plane waves that satisfy suitable requirements concerning wave vectors and the polarization state. ${ }^{1,2}$ The first set of plane waves, usually referred to as ordinary waves, can be expressed as

$$
\begin{aligned}
\mathbf{E}_{o}^{( \pm)} & =\mathbf{U}_{o}^{( \pm)} \exp \left[i \mathbf{k}_{\perp} \cdot \mathbf{r}_{\perp} \pm i z \cdot\left(k_{0}^{2} n_{o}^{2}-k_{\perp}^{2}\right)^{1 / 2}\right] \\
& \equiv \mathbf{U}_{o}^{( \pm)} \exp \left(i \mathbf{k}_{\perp} \cdot \mathbf{r}_{\perp} \pm i k_{o z} z\right),
\end{aligned}
$$


where $\quad \mathbf{k}_{\perp}=k_{x} \hat{\mathbf{e}}_{x}+k_{y} \hat{\mathbf{e}}_{y} \quad$ and $\quad \mathbf{U}_{o}^{( \pm)}=U_{o}^{( \pm)}\left(-k_{y} \hat{\mathbf{e}}_{x}\right.$ $+k_{x} \hat{\mathbf{e}}_{y}$ ), with $U_{o}^{( \pm)}$having two arbitrary constants. The second set, extraordinary waves, can be expressed as

$$
\begin{aligned}
\mathbf{E}_{e}^{( \pm)} & =\mathbf{U}_{e}^{( \pm)} \exp \left[i \mathbf{k}_{\perp} \cdot \mathbf{r}_{\perp} \pm i z \frac{n_{o}}{n_{e}}\left(k_{0}^{2} n_{e}^{2}-k_{\perp}^{2}\right)^{1 / 2}\right] \\
& \equiv \mathbf{U}_{e}^{( \pm)} \exp \left(i \mathbf{k}_{\perp} \cdot \mathbf{r}_{\perp} \pm i k_{e z} z\right),
\end{aligned}
$$

where

$$
\mathbf{U}_{e}^{( \pm)}=U_{e}^{( \pm)}\left[k_{x} \hat{\mathbf{e}}_{x}+k_{y} \hat{\mathbf{e}}_{y}-\frac{n_{o}}{n_{e}} \frac{k_{\perp}^{2}}{\left(k_{o}^{2} n_{e}^{2}-k_{\perp}^{2}\right)^{1 / 2}} \hat{\mathbf{e}}_{x}\right]
$$

and $U_{e}^{( \pm)}$are two arbitrary constants. The vector $\mathbf{k}_{\perp}$ can explore the whole transverse plane. For ordinary waves, the range of values $\left|\mathbf{k}_{\perp}\right| \leqslant k_{0} n_{o}$ is associated with propagating waves, whereas $\left|\mathbf{k}_{\perp}\right|>k_{0} n_{o}$ corresponds to evanescent waves. For extraordinary waves the same holds for $\left|\mathbf{k}_{\perp}\right| \leqslant k_{0} n_{e}$ and $\left|\mathbf{k}_{\perp}\right|>k_{0} n_{e}$, respectively.

The linear nature of Eq. (1) indicates that its most general solution describing forward-propagating fields is conveniently expressed as

$$
\begin{aligned}
\mathbf{E}\left(\mathbf{r}_{\perp}, z\right)= & \int \mathrm{d}^{2} \mathbf{k}_{\perp}\left[\mathbf{U}_{o}^{(+)}\left(\mathbf{k}_{\perp}\right) \exp \left(i \mathbf{k}_{\perp} \cdot \mathbf{r}_{\perp}+i k_{o z} z\right)\right. \\
& \left.+\mathbf{U}_{e}^{(+)}\left(\mathbf{k}_{\perp}\right) \exp \left(i \mathbf{k}_{\perp} \cdot \mathbf{r}_{\perp}+i k_{e z} z\right)\right] \\
\equiv & \mathbf{E}_{o}\left(\mathbf{r}_{\perp}, z\right)+\mathbf{E}_{e}\left(\mathbf{r}_{\perp}, z\right) .
\end{aligned}
$$

Equation (5) provides a fully vectorial angular-spectrum representation of the field and is the starting point of our analysis. By introducing the Fourier transform $\widetilde{\mathbf{E}}\left(\mathbf{k}_{\perp}\right)$ of the electric field on the plane $z=0$

$$
\widetilde{\mathbf{E}}\left(\mathbf{k}_{\perp}\right)=\frac{1}{(2 \pi)^{2}} \int \mathrm{d}^{2} \mathbf{r}_{\perp} \exp \left(-i \mathbf{k}_{\perp} \cdot \mathbf{r}_{\perp}\right) \mathbf{E}\left(\mathbf{r}_{\perp}, 0\right),
$$

one can easily find by imposing the boundary conditions that the constants $U_{o}^{(+)}$and $U_{e}^{(+)}$are related to $\widetilde{\mathbf{E}}\left(\mathbf{k}_{\perp}\right)$ through the relations

$$
\begin{aligned}
& U_{o}^{(+)}\left(\mathbf{k}_{\perp}\right)=-\frac{1}{k_{\perp}^{2}}\left[k_{y} \widetilde{E}_{x}\left(\mathbf{k}_{\perp}\right)-k_{x} \widetilde{E}_{y}\left(\mathbf{k}_{\perp}\right)\right], \\
& U_{e}^{(+)}\left(\mathbf{k}_{\perp}\right)=\frac{1}{k_{\perp}^{2}}\left[k_{x} \widetilde{E}_{x}\left(\mathbf{k}_{\perp}\right)+k_{y} \widetilde{E}_{y}\left(\mathbf{k}_{\perp}\right)\right],
\end{aligned}
$$

which solve, in principle, the problem of propagation inside the crystal. In fact, after the electric field on the plane $z=0$ is known, Eqs. (7), together with Eq. (5), provide the expression of the field in the whole half-space, $z$ $>$ 0. Note that Eqs. (7) contain only the transverse part, $\mathbf{E}_{\perp}=E_{x} \hat{\mathbf{e}}_{x}+E_{y} \hat{\mathbf{e}}_{y}$, of the electric field at $z=0$, in agreement with a general property that states that the $z$ component of the field is known as soon as the transverse part is given (see, e.g., Refs. 6 and 7). Equation (5) shows that an arbitrary field can be expressed as the superposition of two components, an ordinary and an extraordinary one, that propagate independently from each other and that, in general, are both essential for describing propagation.

We wish to note that our formalism can be extended to deal with a propagation direction that is different from that of the $z$ axis. In fact, in the general case, one can obtain the equivalent sets of Eqs. (3) and (4) by performing a coordinate rotation, which transforms the $z$ axis into the new propagation direction, and parametrizing the two sets of plane waves with the new transverse wave vector (which allows the expression of the new longitudinal components $k_{z}$ in terms of $n_{o}$ and $n_{e}$ ).

\section{PARAXIAL THEORY OF PROPAGATION}

By inserting Eqs. (7) into Eq. (5), we obtain

$$
\begin{aligned}
\mathbf{E}_{\perp o}\left(\mathbf{r}_{\perp}, z\right)= & \int \mathrm{d}^{2} \mathbf{k}_{\perp} \exp \left[i \mathbf{k}_{\perp} \cdot \mathbf{r}_{\perp}+i z\left(k_{0}^{2} n_{o}^{2}\right.\right. \\
& \left.\left.-k_{\perp}^{2}\right)^{1 / 2}\right] \mathbf{P}_{o} \cdot \mathbf{E}_{\perp}\left(\mathbf{k}_{\perp}\right), \\
E_{z o}\left(\mathbf{k}_{\perp}, z\right)= & , \\
\mathbf{E}_{\perp e}\left(\mathbf{r}_{\perp}, z\right)= & \int \mathrm{d}^{2} \mathbf{k}_{\perp} \exp \left[i \mathbf{k}_{\perp} \cdot \mathbf{r}_{\perp}+i z \frac{n_{o}}{n_{e}}\left(k_{0}^{2} n_{e}^{2}\right.\right. \\
& \left.\left.-k_{\perp}^{2}\right)^{1 / 2}\right] \mathbf{P}_{e} \cdot \mathbf{E}_{\perp}\left(\mathbf{k}_{\perp}\right), \\
E_{z e}\left(\mathbf{r}_{\perp}, z\right)= & -\frac{n_{o}}{n_{e}} \int \mathrm{d}^{2} \mathbf{k}_{\perp} \exp \left[i \mathbf{k}_{\perp} \cdot \mathbf{r}_{\perp}+i z \frac{n_{o}}{n_{e}}\left(k_{0}^{2} n_{e}^{2}\right.\right. \\
& \left.\left.-k_{\perp}^{2}\right)^{1 / 2}\right] \frac{\mathbf{k}_{\perp} \cdot \mathbf{E}_{\perp}\left(\mathbf{k}_{\perp}\right)}{\left(k_{0}^{2} n_{e}^{2}-k_{\perp}^{2}\right)^{1 / 2}},
\end{aligned}
$$

where the tensors $\mathbf{P}_{o}$ and $\mathbf{P}_{e}$ are defined as

$$
\mathbf{P}_{o}=\frac{1}{k_{\perp}^{2}}\left[\begin{array}{cc}
k_{y}^{2} & -k_{x} k_{y} \\
-k_{x} k_{y} & k_{x}^{2}
\end{array}\right], \quad \mathbf{P}_{e}=\frac{1}{k_{\perp}^{2}}\left[\begin{array}{cc}
k_{x}^{2} & k_{x} k_{y} \\
k_{x} k_{y} & k_{y}^{2}
\end{array}\right] .
$$

The above tensors satisfy the relations $\mathbf{P}_{o}^{2}=\mathbf{P}_{o}, \mathbf{P}_{e}^{2}$ $=\mathbf{P}_{e}, \mathbf{P}_{o}+\mathbf{P}_{e}=1$, and $\mathbf{P}_{o} \cdot \mathbf{P}_{e}=0$ so that they represent a complete set of orthogonal projectors, a property that accounts for the remarkable decomposition of the boundary field $\widetilde{\mathbf{E}}_{\perp}\left(\mathbf{k}_{\perp}\right)$ in an ordinary and an extraordinary part.

The angular spectrum of a paraxial beam is nonnegligible for only $\left|\mathbf{k}_{\perp}\right| \ll k_{0}$. Thus approximate paraxial expressions for the field can be obtained by the expansion of the square roots in Eqs. (8) to the first order in $\left|\mathbf{k}_{\perp}\right|$, which yields

$$
\begin{aligned}
\mathbf{E}_{\perp o}\left(\mathbf{r}_{\perp}, z\right)= & \exp \left(i k_{0} n_{o} z\right) \int \mathrm{d}^{2} \mathbf{k}_{\perp} \\
& \times \exp \left(i \mathbf{k}_{\perp} \cdot \mathbf{r}_{\perp}-\frac{i k_{\perp}^{2}}{2 k_{0} n_{o}} z\right) \mathbf{P}_{o} \cdot \tilde{\mathbf{E}}_{\perp}\left(\mathbf{k}_{\perp}\right) \\
\equiv & \exp \left(i k_{0} n_{o} z\right) \mathbf{A}_{\perp o}\left(\mathbf{r}_{\perp}, z\right), \\
E_{z o}\left(\mathbf{k}_{\perp}, z\right)= & 0, \\
\mathbf{E}_{\perp e}\left(\mathbf{r}_{\perp}, z\right)= & \exp \left(i k_{0} n_{o} z\right) \int \mathrm{d}^{2} \mathbf{k}_{\perp} \\
& \times \exp \left(i \mathbf{k}_{\perp} \cdot \mathbf{r}_{\perp}-\frac{i n_{o} k_{\perp}^{2}}{2 k_{0} n_{e}^{2}} z\right) \mathbf{P}_{e} \cdot \mathbf{E}_{\perp}\left(\mathbf{k}_{\perp}\right) \\
\equiv & \exp \left(i k_{0} n_{o} z\right) \mathbf{A}_{\perp e}\left(\mathbf{r}_{\perp}, z\right),
\end{aligned}
$$




$$
\begin{aligned}
E_{z e}\left(\mathbf{r}_{\perp}, z\right)= & \exp \left(i k_{0} n_{o} z\right)\left(\frac{-n_{o}}{k_{0} n_{e}^{2}}\right) \int \mathrm{d}^{2} \mathbf{k}_{\perp} \\
& \quad \times \exp \left(i \mathbf{k}_{\perp} \cdot \mathbf{r}_{\perp}-\frac{i n_{o} k_{\perp}^{2}}{2 k_{0} n_{e}^{2}} z\right) \mathbf{k}_{\perp} \cdot \tilde{\mathbf{E}}_{\perp}\left(\mathbf{k}_{\perp}\right) \\
\equiv & \exp \left(i k_{0} n_{o} z\right) A_{z e}\left(\mathbf{r}_{\perp}, z\right),
\end{aligned}
$$

where we defined the slowly varying amplitudes A. We note two relevant features of the paraxial field: First, the longitudinal component (along the $z$ axis) is much smaller than the transverse ones because of the smallness of $\left|\mathbf{k}_{\perp}\right| / k_{0}$, a circumstance that is well known in the isotropic case. ${ }^{8}$ Second, the rapidly varying factor is $\exp \left(i k_{0} n_{o} z\right)$ for both the ordinary and the extraordinary waves; this situation follows from the fact that the ordinary and the extraordinary sheets of the normal surface of a uniaxial crystal are tangent in $\mathbf{k}=k_{0} n_{o} \hat{\mathbf{e}}_{z} \cdot{ }^{2}$ The slowly varying amplitudes $\mathbf{A}_{\perp o}\left(\mathbf{r}_{\perp}, z\right)$ and $\mathbf{A}_{\perp e}\left(\mathbf{r}_{\perp}, z\right)$, introduced in Eqs. (10), are easily seen to satisfy the two decoupled parabolic equations

$$
\begin{aligned}
& \left(i \frac{\partial}{\partial z}+\frac{1}{2 k_{0} n_{o}} \nabla_{\perp}^{2}\right) \mathbf{A}_{\perp o}=0, \\
& \left(i \frac{\partial}{\partial z}+\frac{n_{o}}{2 k_{0} n_{e}^{2}} \nabla_{\perp}^{2}\right) \mathbf{A}_{\perp e}=0,
\end{aligned}
$$

where $\nabla_{\perp}=\partial_{x} \hat{\mathbf{e}}_{x}+\partial_{y} \hat{\mathbf{e}}_{y}$. Equations (11) are the anisotropic counterparts of the standard parabolic equation governing paraxial propagation in isotropic media and show how ordinary and extraordinary components propagate independently of each other. In particular, the propagation constants in front of $\nabla_{\perp}^{2}$ are different because they are associated with the respective refractive indices. Thus, besides the standard (Fresnel) behavior exhibited by both components, a paraxial beam possesses diffraction properties that are peculiar to a superposition of (ordinary and extraordinary) waves propagating in different ways.

If we now take advantage of the properties of $\mathbf{P}_{o}$ and $\mathbf{P}_{e}$, we obtain (see Appendix A)

$$
\begin{aligned}
\mathbf{A}_{\perp o}\left(\mathbf{r}_{\perp}, 0\right)= & \frac{1}{2 \pi} \int \mathrm{d}^{2} \mathbf{r}_{\perp}{ }^{\prime} \log \left|\mathbf{r}_{\perp}-\mathbf{r}_{\perp}{ }^{\prime}\right| \\
& \times\left[\begin{array}{cc}
\partial_{y^{\prime}}^{2} & -\partial_{x^{\prime}} \partial_{y^{\prime}} \\
-\partial_{x^{\prime}} \partial_{y^{\prime}} & \partial_{x^{\prime}}^{2}
\end{array}\right] \mathbf{E}_{\perp}\left(\mathbf{r}_{\perp}{ }^{\prime}, 0\right),
\end{aligned}
$$

$$
\begin{aligned}
\mathbf{A}_{\perp e}\left(\mathbf{r}_{\perp}, 0\right)= & \frac{1}{2 \pi} \int \mathrm{d}^{2} \mathbf{r}_{\perp}^{\prime} \log \left|\mathbf{r}_{\perp}-\mathbf{r}_{\perp}{ }^{\prime}\right| \\
& \times\left[\begin{array}{cc}
\partial_{x^{\prime}}^{2} & \partial_{x^{\prime}} \partial_{y^{\prime}} \\
\partial_{x^{\prime}} \partial_{y^{\prime}} & \partial_{x^{\prime}}^{2}
\end{array}\right] \mathbf{E}_{\perp}\left(\mathbf{r}_{\perp}{ }^{\prime}, 0\right),
\end{aligned}
$$

which relate the boundary field $\mathbf{E}_{\perp}\left(\mathbf{r}_{\perp}, 0\right)$ to the ordinary and the extraordinary boundary components $\mathbf{A}_{\perp o}\left(\mathbf{r}_{\perp}, 0\right)$ and $\mathbf{A}_{\perp e}\left(\mathbf{r}_{\perp}, 0\right)$, respectively. Equations (11) and Eqs.

(a)

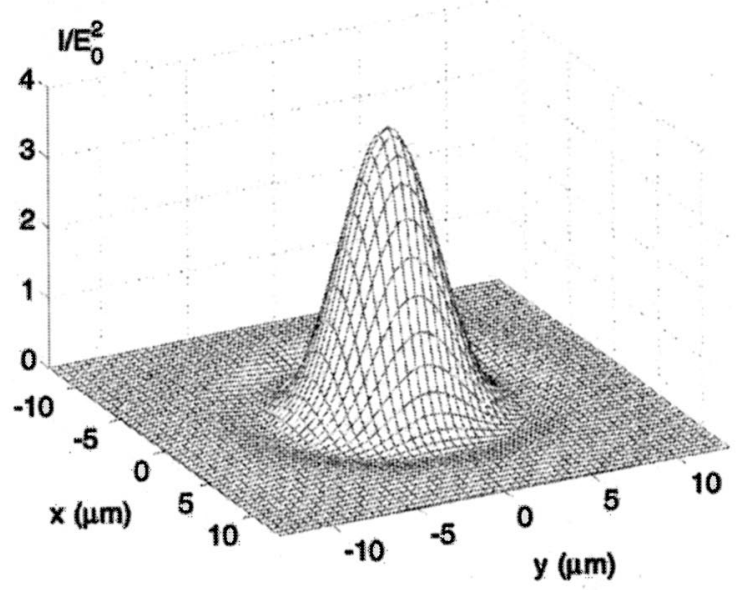

(b)

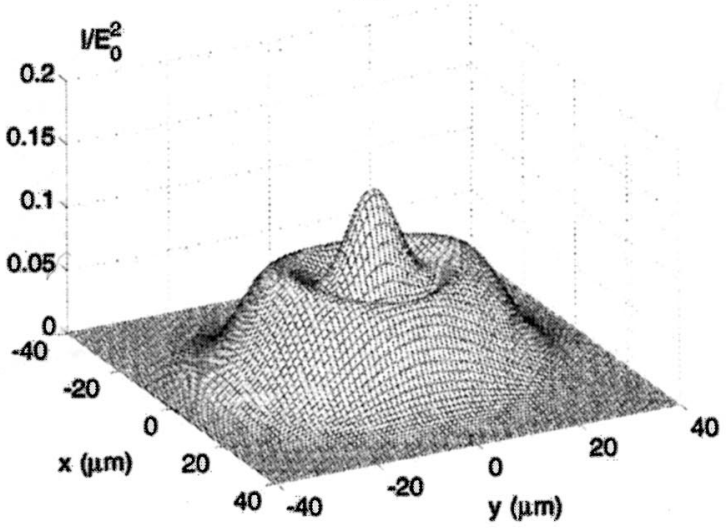

(c)

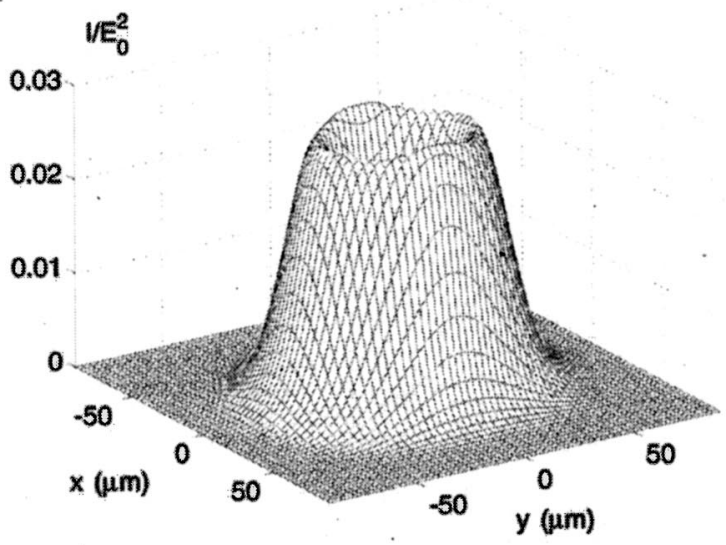

Fig. 1. Plot of the intensity $\left|A_{x}\right|^{2}+\left|A_{y}\right|^{2}$ normalized to $E_{0}^{2}$ for (a) $z=0 \mu \mathrm{m}$, (b) $z=1500 \mu \mathrm{m}$, (c) $z=3000 \mu \mathrm{m}$. The wavelength is $\lambda=0.6328 \mu \mathrm{m}, s=5 \mu \mathrm{m}$, and the crystal is rutile, i.e., $n_{o}=2.616$ and $n_{e}=2.903$. 


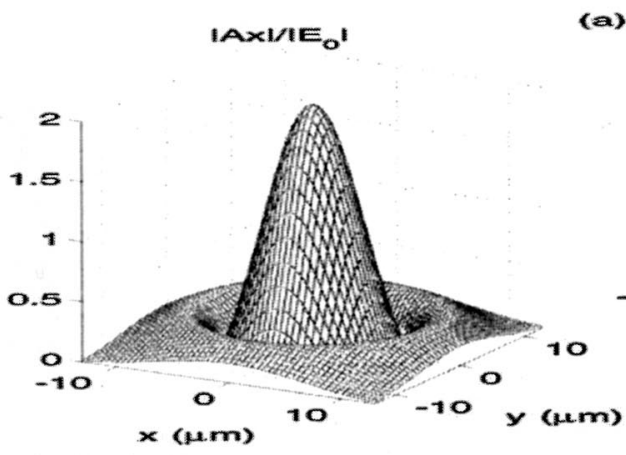

(a)
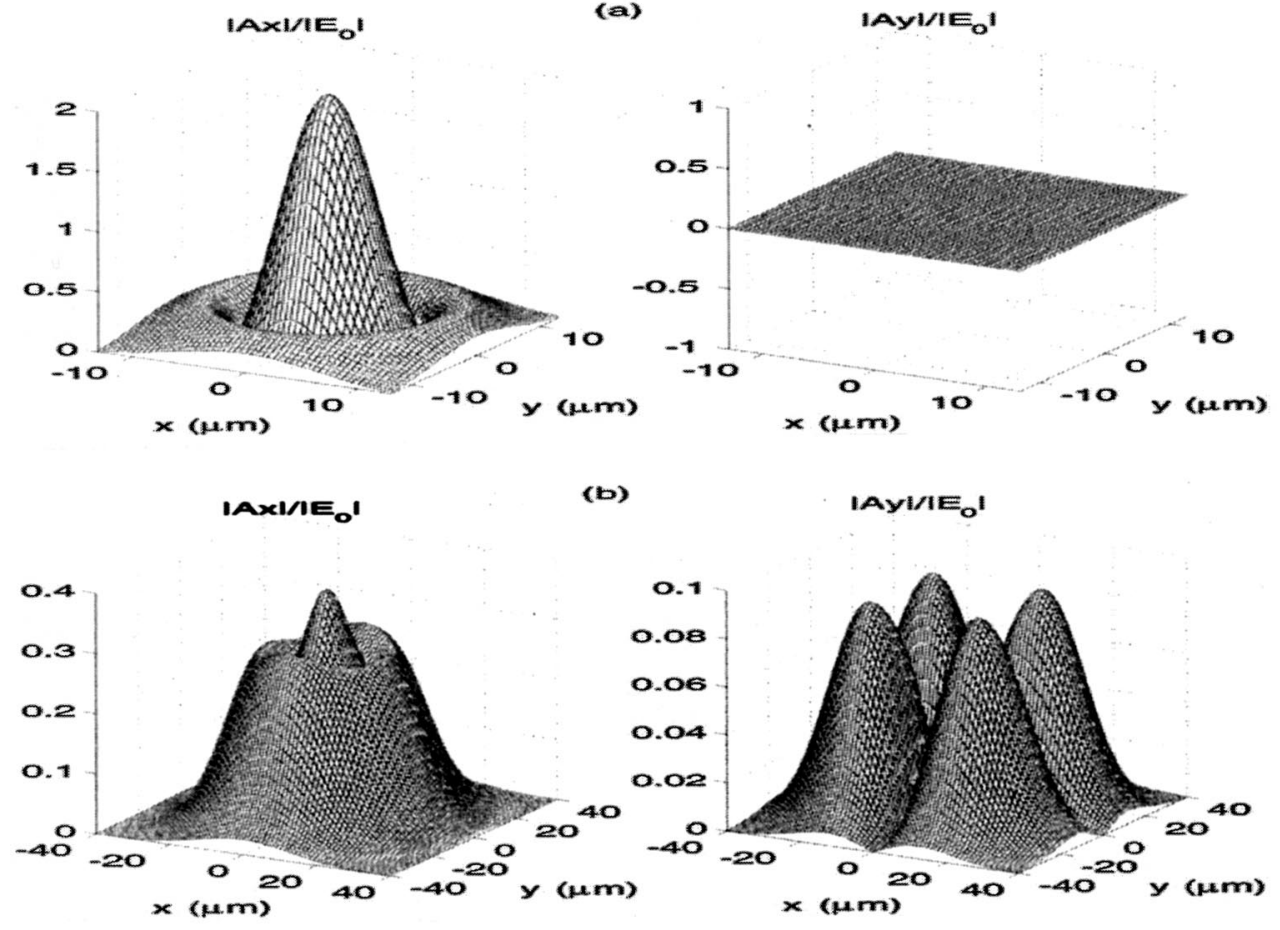

(b) IAYIIE⿸厂⿱
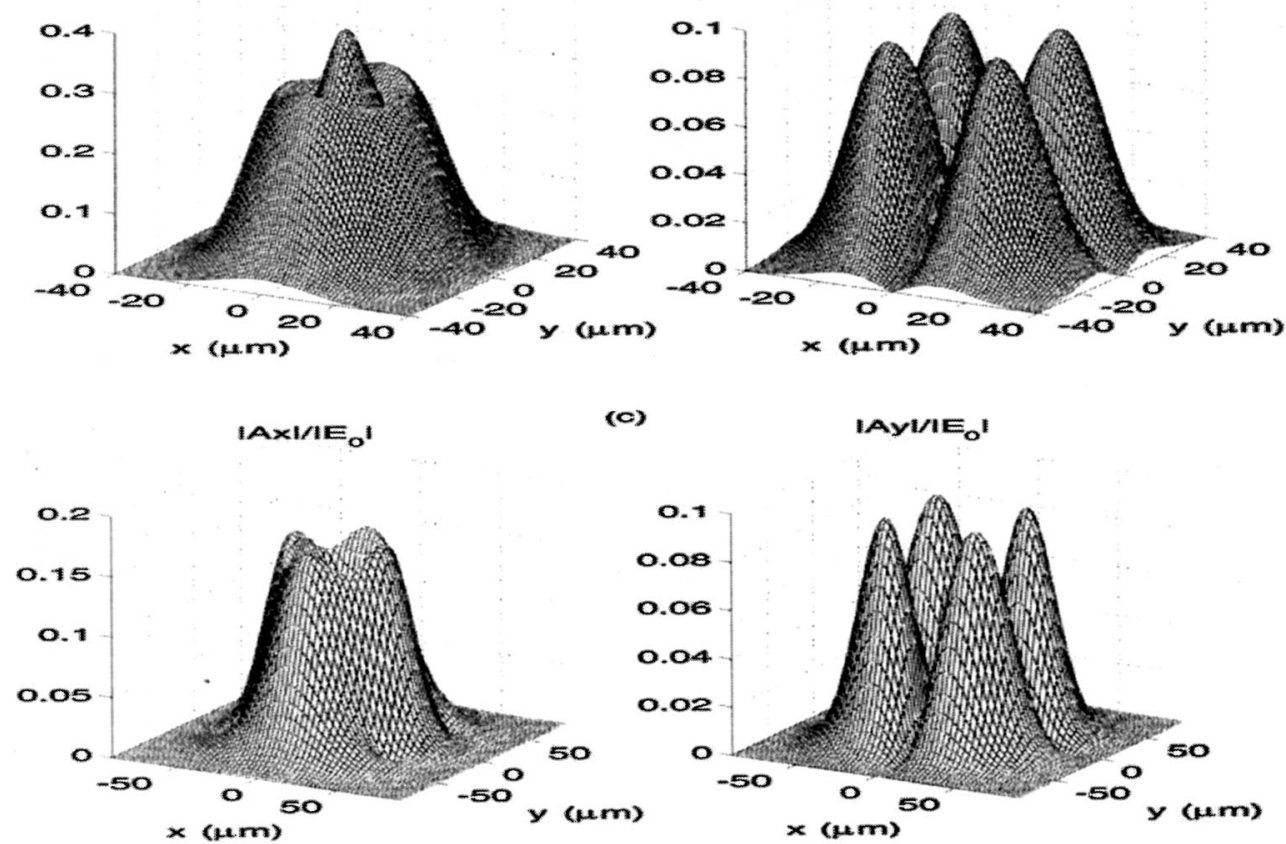

(a)

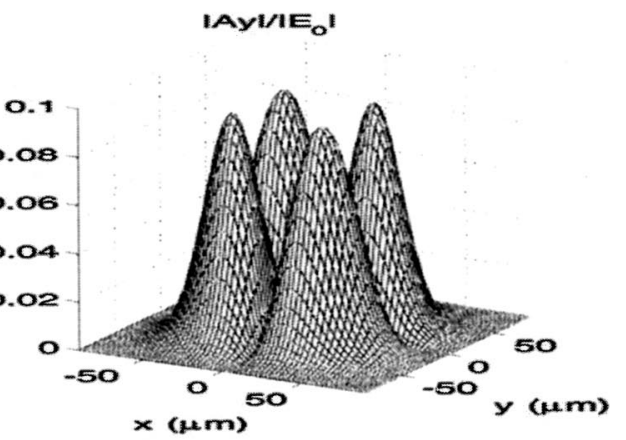

Fig. 2. Plot of the moduli of $A_{x}$ and $A_{y}$ normalized to $\left|E_{0}\right|$ for (a) $z=0 \mu \mathrm{m}$, (b) $z=1500 \mu \mathrm{m}$, (c) $z=3000 \mu \mathrm{m}$.

(12) are the basic relations of our approach. Furthermore, the longitudinal field component is obtained from the transverse ones in the form of (see Appendix B)

$$
A_{z e}\left(\mathbf{r}_{\perp}, z\right)=\frac{i n_{o}}{k_{0} n_{e}^{2}} \nabla_{\perp} \cdot\left[\mathbf{A}_{\perp o}\left(\mathbf{r}_{\perp}, \frac{n_{o}^{2}}{n_{e}^{2}} z\right)+\mathbf{A}_{\perp e}\left(\mathbf{r}_{\perp}, z\right)\right] .
$$

The whole procedure can be summarized as follows: From the knowledge of the boundary transverse field $\mathbf{E}_{\perp}\left(\mathbf{r}_{\perp}, 0\right)$, we obtain through Eqs. (12) the ordinary and the extraordinary boundary amplitudes $\mathbf{A}_{\perp o}\left(\mathbf{r}_{\perp}, 0\right)$ and $\mathbf{A}_{\perp e}\left(\mathbf{r}_{\perp}, 0\right)$, respectively, whose behavior for $z>0$ is obtained by means of Eqs. (11); in turn, the longitudinal component $A_{z e}\left(\mathbf{r}_{\perp}, 0\right)$ for $z \geqslant 0$ is given by Eq. (13). Our approach shows that anisotropic paraxial propagation, unlike that in the isotropic case, cannot be described correctly in a scalar way but requires a fully vectorial treatment.

\section{QUASI-GAUSSIAN VECTORIAL BEAMS}

In this section, we apply our formalism for describing the propagation of the field specified by the boundary condition

$$
\mathbf{E}_{\perp}\left(\mathbf{r}_{\perp}, 0\right)=E_{0}\left(\frac{r_{\perp}^{2}}{s^{2}}-2\right) \exp \left(-\frac{r_{\perp}^{2}}{2 s^{2}}\right) \hat{\mathbf{e}}_{x}
$$

where $E_{0}$ and $s$ are two arbitrary constants that are associated with the intensity and the beam waist, respectively. This quasi-Gaussian field is initially polarized along the $x$ axis, and its initial shape is a Gaussian modulated by the function $\left(r_{\perp}^{2} / s^{2}-2\right)$. Its intensity distribution peaks about the origin and presents a small ring with a radius of $r_{\perp}=2 s$. Instead of applying the general procedure outlined at the end of Section 3, we can more easily treat these beams by directly solving the integrals that appear in Eqs. (10). In fact, the Fourier transform of $\mathbf{E}_{\perp}\left(\mathbf{r}_{\perp}, 0\right)$ is 


$$
\tilde{\mathbf{E}}_{\perp}\left(\mathbf{k}_{\perp}\right)=-E_{0} \frac{s^{4}}{2 \pi} k_{\perp}^{2} \exp \left(-\frac{s^{2} k_{\perp}^{2}}{2}\right) \hat{\mathbf{e}}_{x} .
$$

Equation (15), when inserted into Eqs. (10), yields, after some algebra,

$$
\begin{aligned}
& \mathbf{A}_{\perp \rho}\left(\mathbf{r}_{\perp}, z\right)=\frac{E_{0} s^{4}}{\left(s^{2}+\frac{i z}{k_{0} n_{o}}\right)^{3}}\left\{\left[y^{2}-\left(s^{2}+\frac{i z}{k_{0} n_{o}}\right)\right] \hat{\mathbf{e}}_{x}\right. \\
& \left.+(-x y) \hat{\mathbf{e}}_{y}\right\} \exp \left[\frac{-r_{\perp}^{2}}{2\left(s^{2}+\frac{i z}{k_{0} n_{o}}\right)}\right], \\
& \mathbf{A}_{\perp e}\left(\mathbf{r}_{\perp}, z\right)=\frac{E_{0} s^{4}}{\left(s^{2}+\frac{i n_{o} z}{k_{0} n_{e}^{2}}\right)^{3}}\left\{\left[x^{2}-\left(s^{2}+\frac{i n_{o} z}{k_{0} n_{e}^{2}}\right)\right] \hat{\mathbf{e}}_{x}\right. \\
& \left.+(x y) \hat{\mathbf{e}}_{y}\right\} \exp \left[\frac{-r_{\perp}^{2}}{2\left(s^{2}+\frac{i n_{o} z}{k_{0} n_{e}^{2}}\right)}\right] .
\end{aligned}
$$

In turn, the longitudinal component can be obtained by Eq. (13) and reads

$$
\begin{aligned}
A_{z e}\left(\mathbf{r}_{\perp}, z\right)= & E_{0} s^{4} \frac{i n_{o}}{k_{0} n_{e}^{2}} \frac{x}{\left(s^{2}+\frac{i n_{o} z}{k_{0} n_{e}^{2}}\right)^{4}}\left[4\left(s^{2}+\frac{i n_{o} z}{k_{0} n_{e}^{2}}\right)\right. \\
& \left.-r_{\perp}^{2}\right] \exp \left[\frac{-r_{\perp}^{2}}{2\left(s^{2}+\frac{i n_{o} z}{k_{0} n_{e}^{2}}\right)}\right]
\end{aligned}
$$

Equations (16) and (17) furnish the analytical expression of a paraxial quasi-Gaussian beam. We note that the longitudinal component is negligible with respect to the transverse ones, in agreement with the results of Section 3. In fact, comparing Eqs. (16) with Eq. (17) shows that it is easy to check that the ratio between the transverse and the longitudinal fields is of the order of $k_{0} s$, which is much greater than unity for a paraxial field.

In Figs. 1 and 2, we show plots of the normalized intensity and amplitudes associated with Eqs. (16). In particular, the top of Fig. 1(c) shows an intensity profile that departs from circular symmetry as well as the presence of two lateral lobes, both effects being a consequence of the medium's anisotropy. In fact, ordinary and extraordinary waves propagate with different diffraction lengths $\left(l_{o}=k_{0} n_{o} s^{2} \simeq 648 \mu \mathrm{m}\right.$ and $l_{e}=k_{0} n_{e}^{2} s^{2} / n_{o} \simeq 800 \mu \mathrm{m}$, respectively), and this difference is responsible for a mutual sliding of the two waves, leading to the observed effects. Furthermore, the initial $x$-axis polarization (apart from the unavoidable small $z$ component associated with the beam's vectorial nature) is not preserved during propagation (see Fig. 2) because of the onset of an increasing $y$ component of the field, this also being related to the different diffraction lengths. The complete evolution of the state of polarization can be obtained from Eqs. (16) and turns out to be a complicated function of the propagation distance $z$.

\section{CONCLUSIONS}

We have investigated the paraxial vectorial propagation of monochromatic waves in uniaxially anisotropic crystals. We have shown that the transverse parts of ordinary and extraordinary waves obey two uncoupled parabolic equations that can be solved in the standard way after the transverse input field is specified on a given plane, $z=0$. The longitudinal part of the extraordinary waves is expressible in terms of the transverse field (that of the ordinary wave being zero). By specifying the input field as a quasi-Gaussian linearly polarized field, we were able to derive exact analytic solutions that reveal the main features of paraxial vector propagation in uniaxial crystals.

\section{APPENDIX A: DECOMPOSITION OF THE BOUNDARY FIELD $E_{\perp}\left(r_{\perp}, 0\right)$ INTO THE ORDINARY AND THE EXTRAORDINARY COMPONENTS}

Let us consider the expression for the ordinary component of the field on the plane $z=0$ :

$$
\begin{aligned}
\mathbf{A}_{\perp o}\left(\mathbf{r}_{\perp}, 0\right)= & \int \mathrm{d}^{2} \mathbf{k}_{\perp} \exp \left(i \mathbf{k}_{\perp} \cdot \mathbf{r}_{\perp}\right) \frac{1}{k_{\perp}^{2}}\left[\begin{array}{cc}
k_{y}^{2} & -k_{x} k_{y} \\
-k_{x} k_{y} & k_{x}^{2}
\end{array}\right] \\
& \times\left[\begin{array}{c}
\widetilde{E}_{x}\left(\mathbf{k}_{\perp}\right) \\
\widetilde{E}_{y}\left(\mathbf{k}_{\perp}\right)
\end{array}\right] .
\end{aligned}
$$

By taking the $\nabla_{\perp}^{2}$ of both sides of Eq. (A1), we obtain

$$
\begin{aligned}
\nabla_{\perp}^{2} \mathbf{A}_{\perp o}\left(\mathbf{r}_{\perp}, 0\right)= & -\int \mathrm{d}^{2} \mathbf{k}_{\perp} \exp \left(i \mathbf{k}_{\perp} \cdot \mathbf{r}_{\perp}\right)\left[\begin{array}{cc}
k_{y}^{2} & -k_{x} k_{y} \\
-k_{x} k_{y} & k_{x}^{2}
\end{array}\right] \\
& \times\left[\begin{array}{c}
\widetilde{E}_{x}\left(\mathbf{k}_{\perp}\right) \\
\widetilde{E}_{y}\left(\mathbf{k}_{\perp}\right)
\end{array}\right] \\
= & {\left[\begin{array}{cc}
\partial_{y}^{2} & -\partial_{x} \partial_{y} \\
-\partial_{x} \partial_{y} & \partial_{x}^{2}
\end{array}\right] \int \mathrm{d}^{2} \mathbf{k}_{\perp} \exp \left(i \mathbf{k}_{\perp} \cdot \mathbf{r}_{\perp}\right) } \\
& \times\left[\begin{array}{c}
\widetilde{E}_{x}\left(\mathbf{k}_{\perp}\right) \\
\widetilde{E}_{y}\left(\mathbf{k}_{\perp}\right)
\end{array}\right] \\
= & {\left[\begin{array}{cc}
\partial_{y}^{2} & -\partial_{x} \partial_{y} \\
-\partial_{x} \partial_{y} & \partial_{x}^{2}
\end{array}\right] \mathbf{E}_{\perp}\left(\mathbf{r}_{\perp}, 0\right) . }
\end{aligned}
$$


Equation (A2) is a standard vectorial two-dimensional Poisson equation whose solution, if its right-hand side is assumed to be square integrable, is given by ${ }^{9}$

$$
\begin{aligned}
\mathbf{A}_{\perp o}\left(\mathbf{r}_{\perp}, 0\right)= & \frac{1}{2 \pi} \int \mathrm{d}^{2} \mathbf{r}_{\perp}{ }^{\prime} \log \left|\mathbf{r}_{\perp}-\mathbf{r}_{\perp}{ }^{\prime}\right| \\
& \times\left[\begin{array}{cc}
\partial_{y^{\prime}}^{2} & -\partial_{x^{\prime}} \partial_{y^{\prime}} \\
-\partial_{x^{\prime}} \partial_{y^{\prime}} & \partial_{x^{\prime}}^{2}
\end{array}\right] \mathbf{E}_{\perp}\left(\mathbf{r}_{\perp}{ }^{\prime}, 0\right),
\end{aligned}
$$

which coincides with Eq. (12a). An analogous calculation can be carried out for the extraordinary part of the field, yielding Eq. (12b).

\section{APPENDIX B: LONGITUDINAL COMPONENT OF THE FIELD IN TERMS OF THE TRANSVERSE PART}

The expression for the longitudinal component of the field in paraxial approximation reads

$$
\begin{aligned}
A_{z e}\left(\mathbf{r}_{\perp}, z\right)= & -\frac{n_{o}}{k_{0} n_{e}^{2}} \int \mathrm{d}^{2} \mathbf{k}_{\perp} \exp \left(i \mathbf{k}_{\perp} \cdot \mathbf{r}_{\perp}-\frac{i n_{o} k_{\perp}^{2}}{2 k_{0} n_{e}^{2}} z\right) \mathbf{k}_{\perp} \\
& \cdot \widetilde{\mathbf{E}}_{\perp}\left(\mathbf{k}_{\perp}\right),
\end{aligned}
$$

[see Eq. (10d)], which can easily be rewritten as

$$
\begin{aligned}
A_{z e}\left(\mathbf{r}_{\perp}, z\right)= & \frac{i n_{o}}{k_{0} n_{e}^{2}} \nabla_{\perp} \cdot \int \mathrm{d}^{2} \mathbf{k}_{\perp} \\
& \times \exp \left(i \mathbf{k}_{\perp} \cdot \mathbf{r}_{\perp}-\frac{i n_{o} k_{\perp}^{2}}{2 k_{0} n_{e}^{2}} z\right) \widetilde{\mathbf{E}}_{\perp}\left(\mathbf{k}_{\perp}\right),
\end{aligned}
$$

By inserting the completeness relation, $\mathbf{P}_{o}+\mathbf{P}_{e}=1$, inside the integral immediately on the left-hand side of the term $\widetilde{\mathbf{E}}_{\perp}\left(\mathbf{k}_{\perp}\right)$, we obtain

$$
\begin{aligned}
A_{z e}\left(\mathbf{r}_{\perp}, z\right)= & \frac{i n_{o}}{k_{0} n_{e}^{2}} \nabla_{\perp} \cdot \int \mathrm{d}^{2} \mathbf{k}_{\perp} \\
& \times \exp \left(i \mathbf{k}_{\perp} \cdot \mathbf{r}_{\perp}-\frac{i k_{\perp}^{2}}{2 k_{0} n_{o}} \frac{n_{o}^{2}}{n_{e}^{2}} z\right) \mathbf{P}_{o} \\
& \cdot \widetilde{\mathbf{E}}_{\perp}\left(\mathbf{k}_{\perp}\right)+\frac{i n_{o}}{k_{0} n_{e}^{2}} \nabla_{\perp} \cdot \int \mathrm{d}^{2} \mathbf{k}_{\perp} \\
& \times \exp \left(i \mathbf{k}_{\perp} \cdot \mathbf{r}_{\perp}-\frac{i n_{o} k_{\perp}^{2}}{2 k_{0} n_{e}^{2}} z\right) \mathbf{P}_{e} \cdot \widetilde{\mathbf{E}}_{\perp}\left(\mathbf{k}_{\perp}\right) \\
= & \frac{i n_{o}}{k_{0} n_{e}^{2}} \nabla_{\perp}\left[\mathbf{A}_{\perp o}\left(\mathbf{r}_{\perp}, \frac{n_{o}^{2}}{n_{e}^{2}} z\right)+\mathbf{A}_{\perp e}\left(\mathbf{r}_{\perp}, z\right)\right]
\end{aligned}
$$

Equation (B3) coincides with Eq. (13).

Direct correspondence to A. Ciattoni at the address on the title page or e-mail, alessandro.ciattoni@ aquila.infn.it.

\section{REFERENCES AND NOTES}

1. M. Born and E. Wolf, Principles of Optics (Pergamon, Oxford, UK, 1999).

2. A. Yariv and P. Yeh, Optical Waves in Crystals (Wiley, New York, 1984).

3. A. Yariv, Optical Electronics (Holt, Rinehart \& Winston, New York, 1985).

4. P. Yeh, Introduction to Photorefractive Nonlinear Optics (Wiley, New York, 1993).

5. J. J. Stamnes and G. C. Sherman, "Radiation of electromagnetic fields in uniaxially anisotropic media," J. Opt. Soc. Am. 66, 780-788 (1976).

6. A. Ciattoni, B. Crosignani, and P. Di Porto, "Vectorial freespace optical propagation: a simple approach for generating all-order nonparaxial corrections," Opt. Commun. 177, 9-13 (2000).

7. A. Ciattoni, P. Di Porto, B. Crosignani, and A. Yariv, "Vectorial nonparaxial propagation equation in the presence of a tensorial refractive-index perturbation," J. Opt. Soc. Am. B17, 809-819 (2000).

8. M. Lax, W. H. Louisell, and W. B. McKnight, "From Maxwell to paraxial wave optics," Phys. Rev. A 11, 1365-1370 (1975).

9. See, for example, G. Arfken, Mathematical Methods for Physicists (Academic, New York, 1984), Sec. 16.6. 